\title{
Comparison of gastric juice soluble triggering receptor expressed on myeloid cells and C-reactive protein for detection of Helicobacter pylori infection
}

\author{
Ahmad Piroozmand ${ }^{1,2}$, Babak Soltani ${ }^{3,4}$, Mohsen Razavizadeh ${ }^{5}$, Amir Hasan Matini ${ }^{6}$, Gholam Abbas Moosavi ${ }^{7}$, \\ Mohammad Salehi ${ }^{8}$, Siamak Soltani ${ }^{9}$
}

${ }^{1}$ Ph.D. of Virology, Associate Professor, Department of Microbiology, Faculty of Medicine, Kashan University of Medical Sciences, Kashan, Iran

${ }^{2}$ Autoimmune Diseases Research Center, Kashan University of Medical Sciences, Kashan, Iran

${ }^{3}$ M.D., Pediatric Infectious Diseases Specialist, Associate Professor, Department of Pediatrics, Faculty of Medicine, Kashan University of Medical Sciences, Kashan, Iran

${ }^{4}$ Infectious Diseases Research Center, Kashan University of Medical Sciences, Kashan, Iran

${ }^{5}$ M.D., Gastroenterologist, Assistant Professor, Department of Internal Medicine, Faculty of Medicine, Kashan University of Medical Sciences, Kashan, Iran

${ }^{6}$ M.D., Pathologist, Assistant Professor, Department of Pathology, Faculty of Medicine, Kashan University of Medical Sciences, Kashan, Iran

${ }^{7}$ M.Sc. of Biostatistics, Lecturer, Department of Public Health, Faculty of Health, Kashan University of Medical Sciences, Kashan, Iran

${ }^{8}$ M.D., Student Research Committee, Kashan University of Medical Sciences, Kashan, Iran

${ }^{9}$ M.D., Forensic Medicine Specialist, Associate Professor, Department of Forensic Medicine, Faculty of Medicine, Iran University of Medical Sciences, Tehran, Iran

Type of article: Original

\begin{abstract}
Background and aim: Triggering receptor expressed on myeloid cells (TREM-1) is a receptor on phagocytes that is triggered by infectious agents. The soluble form of it (sTREM-1) can be elevated in gastric juice by Helicobacter pylori (H. pylori) infection of gastric mucosa. The aim of this study was to compare the diagnostic values of sTREM-1 and C-reactive protein (CRP) for detection of $H$. pylori infection in gastric mucosa.

Methods: In this diagnostic accuracy study on cases who underwent endoscopy from March 2015 to July 2016 in Shahid Beheshti Hospital, Kashan, Iran, gastric juice sTREM-1 and CRP concentrations were measured by enzyme-linked immunosorbent assays (ELISA) and their diagnostic values were compared to detect $H$. pylori infection. Gold standard test was histopathology. Data were entered into SPSS software version 16. Statistical analysis was made by Kolmogorov-Smirnov, Chi-square, Independent-samples t-test, Kruskal-Wallis, MannWhitney U, Pearson product-moment correlation, Receiver operating characteristic curve (ROC), Brier score, Nagelkerke R square and scaled reliability test.

Results: Of a total of 160 cases, 81 (50.6\%) were $H$. pylori-positive based on pathology. The level of sTREM-1 in $H$. pylori-positive patients was significantly higher than $H$. pylori-negative patients $(\mathrm{p}=0.000)$, but no significant difference between CRP concentrations was shown between groups $(\mathrm{p}=0.7)$. Sensitivity, specificity, positive likelihood ratio, negative likelihood ratio and accuracy of sTREM-1 were $82 \%, 75 \%, 3.3,0.25,78 \%$ and for CRP were $62 \%, 40 \%, 1.02,0.98,51 \%$ respectively for diagnosis of $H$. pylori infection. True positive and negative rates were $66(81.5 \%)$ and $59(74.7 \%)$ for sTREM-1 and $50(61.7 \%)$ and $31(39.2 \%)$ for CRP. The levels of sTREM-1 and CRP were not significantly different between endoscopic finding groups $(\mathrm{p}=0.97, \mathrm{p}=0.2$ respectively).
\end{abstract}

Conclusion: Despite CRP, sTREM-1 was a relatively acceptable indicator of $H$. pylori infection of gastric mucosa.

Keywords: sTREM-1, CRP, Helicobacter pylori, Gastric ulcer, Duodenal ulcer, Gastritis

\section{Corresponding author:}

Associate Professor Dr. Babak Soltani, Kashan University of Medical Sciences, Kashan, Iran.

Tel: +98.9123887085, Fax: +98.3155548900, Email: babak_soltani1969@yahoo.com

Received: May 04, 2017, Accepted: October 20, 2017, Published: December 2017

iThenticate screening: October 22, 2017, English editing: October 26, 2017, Quality control: December 12, 2017

This article has been reviewed / commented by three experts

(C) 2018 The Authors. This is an open access article under the terms of the Creative Commons Attribution-NonCommercialNoDerivs License, which permits use and distribution in any medium, provided the original work is properly cited, the use is non-commercial and no modifications or adaptations are made. 


\section{Introduction}

The exact mechanisms of peptic ulcer disease (gastric and duodenal ulcers) are not clear (1). Many investigations have been noted about impairment of repair and protection of gastric and duodenal mucosa (2). It seems most cases of peptic ulcer disease occur in presence of Helicobacter pylori (H. pylori) infection. Evidence is supported that $H$. pylori is a necessary part of ulcerations such as acid and pepsin. During a study, it was shown that $H$. pylori secreted the lipopolysaccharide (LPS) which contributed to the gastric inflammation (3). Indeed, it is unclear whether the inflammation or the micro-organism is the mainstay cause of ulcerative process (4). Triggering receptor expressed on myeloid cells (TREM-1) is a receptor that is expressed on the surface of monocytes and neutrophils in acute inflammatory processes such as sepsis, and is increased by bacteria and fungus $(5,6)$. Its soluble form (sTREM-1) is shown in the gastric juice of cases with peptic ulcer disease and gastritis (7-9). According to some investigations, the diagnostic value of sTREM-1for detection of bacterial infections is unclear (10-13). An investigation indicated the association of gastric juice sTREM-1 level with gastric $H$. pylori infection (9), but in another study, no association was found (7). C-reactive protein (CRP) is a useful marker for diagnosis of inflammation and sepsis in children and neonates. It is produced in the liver by stimulation of IL-6 (14). Some surveys demonstrate a good correlation between serum and salivary CRP levels for detection of infection $(15,16)$. Another study showed low diagnostic values of CRP in prediction of infection (17). During a survey, a high level of serum CRP was detected among $H$. pylori infected patients (18), meanwhile in another study, no increase in serum CRP level was found in $H$. Pylori infected cases (19). Despite serum CRP, Gastric juice CRP concentration has not been measured for detection of $H$. pylori infection in any study. Based on the above contradictions regarding diagnostic values of sTREM-1 and CRP in detection of infection, the present study was conducted to compare the diagnostic values of sTREM-1 and CRP for detection of gastric $H$. pylori infection. Despite these biomarkers, other diagnostic modalities such as stool antigen test and PCR are expensive, and H. pylori IgG may remain positive for long time after successful treatment. So, positive results of our tests may indicate active disease. Furthermore, if an acceptable diagnostic value of these indicators will be approved in the future, their levels in gastric juice by nasogastric tube (NG tube) suction may be useful as a screening test for detection of $H$. pylori infection because it is a less invasive procedure than endoscopy.

\section{Material and Methods}

\subsection{Study population and design}

This diagnostic accuracy study was carried out on patients who were candidates for endoscopy from March 2015 to July 2016 in Shahid Beheshti Hospital of Kashan. A total of 160 cases participated in present investigation. Indications for endoscopy were nausea and vomiting accompanied by epigastric pain, abdominal discomfort or pain and dyspepsia.

\subsection{Selection criteria}

\subsubsection{Inclusion criteria}

Inclusion criteria included individuals aged 18 years or older who were referred for endoscopy. Exclusion criteria were age less than 18 years, acute or chronic renal failure, recent upper gastrointestinal (GI) bleeding, chronic liver disease, diabetes mellitus, gastric carcinoma, intake of any antibiotics, proton pump inhibitors (PPIs), bismuth, nonsteroidal anti-inflammatory drugs (NSAIDs) or corticosteroids during recent 2 weeks.

\subsubsection{Exclusion criteria}

In all, 31 patients were excluded before beginning the study ( 21 cases used PPIs, 5 cases used antibiotics and 5 cases refused to undergo endoscopy). After enrollment, no bodies were excluded from the study. The patients were selected regarding convenient sample strategy.

\subsection{Sample size}

Sample size was estimated according to $\alpha=0.05, \beta=0.2, \mu_{1}=150.14, \mu_{2}=118.48, \mathrm{SD}_{1}=41.16, \mathrm{SD}_{2}=54.93$ (9). It was calculated as about 80 cases.

\subsection{Data collection and Laboratory methods}

According to biopsy samples, patients were divided into two groups (H. pylori-positive and H. pylori-negative groups). Gastric juices were collected for measurement of sTREM-1 and CRP levels, and their diagnostic values were compared together for detection of $H$. pylori infection regarding histopathology (gold standard test). The interval between gastric juice sampling and performance of ELISA tests was about 6 months in two steps (over approximately 12 months). The histopathology test was done up to a maximum of 2 weeks. Endoscopy was done by a physician. Endoscopic findings were based on macroscopic findings such as normal endoscopy, gastric ulcer (GU), duodenal ulcer (DU) and gastritis. About 5-7 ml of gastric juice samples were aspirated and transferred to a 
laboratory at Shahid Beheshti Hospital of Kashan, and were frozen and stored at $-20^{\circ} \mathrm{C}$ until processing for measurement of sTREM-1 and CRP concentrations. Laboratory tests were conducted by a technician. After thawing gastric juices, they were centrifuged at 2000 r.p.m. for twenty minutes and the supernatants were removed, then sTREM-1 (Hangzhou Eastbiopharm, China) and CRP (R\&D system, CRP Quantikine, USA) were measured by enzyme-linked immunosorbent assay (ELISA) kits. For estimation of sTREM-1, captured antibody of sTREM-1 was diluted to $4000 \mathrm{ng} / \mathrm{ml}$ and with $0.1 \mathrm{ml}$ per well, distributed in a 96-well plate. Supernatants of gastric juices were incubated overnight at $25{ }^{\circ} \mathrm{C}$, wells were washed with $0.05 \%$ solution of phosphate-buffered saline with pH: 7.2-7.4 and $0.1 \mathrm{ml}$ standard concentrations of sTREM-1 $(15.1-4000 \mathrm{pg} / \mathrm{ml})$ diluted with reagent solution and added to wells. Following 2 hours of incubation, the wells were washed three times and $0.1 \mathrm{ml}$ of $400 \mathrm{ng} / \mathrm{ml}$ dilution of sTREM-1 detection antibody was added to wells. After incubation for 2 hours, attached antibodies were signaled by streptavidin. Concentrations of sTREM-1 were calculated by optical density of $450 \mathrm{~nm}$ following addition of 1:1 $\mathrm{H} 2 \mathrm{O} 2$ : tetramethylbenzidine as a substrate. The lowest level of detection for sTREM-1 was $5 \mathrm{pg} / \mathrm{ml}$. For estimation of CRP, $100 \mu \mathrm{l}$ of assay diluent was added to each well. Then $50 \mu \mathrm{l}$ of gastric supernatant was added per well and incubated for 2 hours. Aspiration was done, and washing of each well was performed 3 times. Two hundred micro liters of conjugate solution were added to each well and incubated for 2 hours. Aspiration was conducted and washing was done 4 times. Thereafter, $200 \mu \mathrm{l}$ of substrate solution was added to each well and incubated for 30 minutes. Finally, $50 \mu \mathrm{l}$ of stop solution was added and the CRP levels were calculated at optical density of $450 \mathrm{~nm}$ within 30 minutes. The lowest limit of detection for CRP was $0.8 \mathrm{ng} / \mathrm{ml}$. Validation of ELISA kits was tested by undiluted calibrators which were made by the manufacturer, and finally two calibration plots were designed.

\subsection{Histopathology}

Two biopsy samples were obtained from corpus and gastric antrum, and were embedded in $10 \%$ formalin and stained by hematoxylin-eosin and Giemsa to detect histopathologic changes. Immunochemistry (gold standard test) was conducted for detection of $\mathrm{H}$. pylori with 1/100 dilution (Biocare Med., California, USA). One pathologist evaluated the histopathology of samples.

\subsection{Statistical analysis}

Data were entered into SPSS software version 16 (Chicago, Illinois, USA). Kolmogorov-Smirnov test was used to determine the normal distribution of continuous data. Continuous data with normal distribution was shown by mean \pm standard deviation (SD), and continuous data with abnormal distribution was reported as median \pm inter quartile range (IQR). Categorical variables were determined by frequency and percent. Concentrations of sTREM-1 and CRP were indicated by median $\pm \mathrm{IQR}$ and mean $\pm \mathrm{SD}$ respectively. Comparison of sTREM-1 and CRP concentrations between groups ( $H$. pylori-negative and H. pylori-positive groups) and sexes was made by MannWhitney $U$ and Independent-samples t-test respectively. Concentrations of sTREM-1 and CRP according to endoscopic findings, were compared by Kruskal-Wallis and one-way ANOVA tests respectively. Association of endoscopic findings and sex with $H$. pylori infection was detected by Chi-square test. The relation between age, sTREM-1 and CRP levels was indicated by Pearson product-moment correlation. The association between H. pylori infection and age was indicated by Independent-samples t-test. Receiver operating characteristic curve (ROC) detected the cut-off values, areas under the curves (AUCs), sensitivities and specificities of diagnostic tests. For optimization of cut-off points, Youden's J statistic index was used. Cut-off values of sTREM-1 and CRP were chosen $16.5 \mathrm{pg} / \mathrm{ml}$ and $4.9 \mathrm{ng} / \mathrm{ml}$ respectively. The overall performance of two biomarkers was evaluated by Brier score, Nagelkerke R square and scaled reliability test. Any $\mathrm{p}<0.05$ was considered statistically significant.

\subsection{Research ethics}

The present study was approved by the ethics committee of the Deputy of Research, Kashan University of Medical Sciences (No. 29/5/1/5022). The informed written consent was obtained from all patients, and their data were noted in questionnaires. All individuals were entered voluntarily in the study. Dangers and benefits of the procedures were described completely for the patients. To decrease the anticipated complications, an expert anesthesiologist and endoscopist were present during the procedure. Moreover, a complete resuscitation set was at hand. Any patient was free to resign from the study at any time. The information of the cases was kept confidential. No charge was imposed to any patient.

\section{Results}

A total of 160 patients, $76(47.5 \%)$ females and $84(52.5 \%)$ males with mean age of $41.07 \pm 15.2$ years (age range of 19-89 years) were entered into this study. A total of 81 cases $(50.6 \%)$ were $H$. pylori-positive. Endoscopic findings were reported as: 22 (13.8\%) normal, 35 (21.9\%) gastric ulcer, 70 (43.8\%) duodenal ulcer and 33 (20.6\%) gastritis. 
Table 1 indicates the association of some variables with $H$. pylori infection. It shows a significant association between sTREM-1 level and $H$. pylori infection $(\mathrm{p}=0.000)$, but no association between CRP level and $H$. pylori infection $(p=0.7)$. No sex predominance was found according to sTREM-1 $(p=0.1)$ and CRP $(p=0.3)$ levels. The relation between age and levels of sTREM-1 $(\mathrm{p}=0.9, \mathrm{r}=-0.01)$ and CRP $(\mathrm{p}=0.4, \mathrm{r}=0.06)$ was not significant. Table 2 indicates the diagnostic values of sTREM-1 and CRP for detection of $H$. pylori infection. The AUCs were 0.86 (95\% CI: $0.81-0.92, \mathrm{p}=0.000)$ and $0.49(95 \%$ CI: $0.40-0.58, \mathrm{p}=0.87)$ for sTREM-1 and CRP respectively (Figure 1 shows ROC curve). For sTREM-1, true negative (TN), false negative (FN), false positive (FP) and true positive (TP) rates were $59(74.7 \%), 15(18.5 \%), 20(25.3 \%)$ and $66(81.5 \%)$ respectively, and for CRP, TN, FN, FP and TP rates were $31(39.2 \%), 31(38.3 \%), 48(60.8 \%)$ and $50(61.7 \%)$ respectively. Brier score was 0.05 and 0.26 and Nagelkerke R square was 0.53 and 0.001 for sTREM-1 and CRP respectively. Scaled reliability (Cronbach's Alpha) between STREM-1 and CRP was 0.005 that showed unreliability of CRP for detection of H. pylori. Finally, despite CRP, sTREM-1 was a relatively acceptable diagnostic test for detection of $H$. pylori infection. Table 3 depicts concentrations of sTREM-1 and CRP according to endoscopic findings that show no differences between them $(\mathrm{p}=0.97, \mathrm{p}=0.2$ respectively). Calibration plots for ELISA kits are depicted by Figures 2 and 3 .

Table 1. Association of variables with Helicobacter pylori infection

\begin{tabular}{|l|l|l|l|l|}
\hline Variables & H. pylori + ; 81 (50.6) & H. pylori - ; 79 (49.4) & p-value \\
\hline \multirow{2}{*}{ Sex, n (\%) } & Male & $39(46.4)$ & $45(53.6)$ & 0.26 \\
\cline { 2 - 5 } & Female & $42(55.3)$ & $34(44.7)$ & \\
\hline Age (years); Mean (SD) & & $42.2(14.6)$ & $39.9(15.8)$ & 0.36 \\
\hline \multirow{3}{*}{ Endoscopy, n (\%) } & Normal & $70(31.8)$ & $15(68.2)$ & 0.2 \\
& Gastric ulcer & $21(60)$ & $14(40)$ & \\
\cline { 2 - 5 } & Duodenal ulcer & $36(51.4)$ & $34(48.6)$ & \\
\cline { 2 - 5 } & Gastritis & $17(51.5)$ & $16(48.5)$ & \\
\hline CRP (ng/ml); Mean (SD) & & $6.08(2.9)$ & $6.3(3.3)$ & 0.7 \\
\hline sTREM-1 (pg/ml); Median (IQR) & & $39(22-75)$ & $12(7-17)$ & 0.000 \\
\hline
\end{tabular}

H. pylori,+ Helicobacter pylori-positive; H. pylori -, Helicobacter pylori-negative; SD, standard deviation; IQR, inter quartile range; CRP, C-reactive protein; sTREM-1, soluble triggering receptor expressed on myeloid cells.

Table 2. Diagnostic values of sTREM-1 and CRP for detection of $H$. pylori infection

\begin{tabular}{|l|l|l|}
\hline Diagnostic values & sTREM-1 & CRP \\
\hline Sensitivity (95\% CI) & $82 \%(71.30-89.25)$ & $62 \%(50.26-72.31$ \\
\hline Specificity (95\% CI) & $75 \%(63.64-83.80)$ & $40 \%(28.44-50.87)$ \\
\hline Positive Predictive Value (95\% CI) & $77 \%(69.02-83.01)$ & $51 \%(44.87-57.14)$ \\
\hline Negative Predictive Value $(95 \% \mathrm{CI})$ & $80 \%(70.99-86.34)$ & $50 \%(40.38-59.62)$ \\
\hline LR- $(95 \%$ CI) & $0.25(0.015-0.40)$ & $0.98(0.66-1.44)$ \\
\hline LR+ $95 \%$ CI) & $3.3(2.17-4.77)$ & $1.02(0.79-1.30)$ \\
\hline Accuracy & $78 \%$ & $51 \%$ \\
\hline
\end{tabular}

sTREM-1, soluble triggering receptor expressed on myeloid cells; CRP, C-reactive protein; H. pylori, Helicobacter pylori; LR-, negative likelihood ratio; LR+, positive likelihood ratio; CI, confidence interval

Table 3. Concentrations of sTREM-1 and CRP according to endoscopic findings

\begin{tabular}{|l|l|l|l|l|l|}
\hline Concentrations & Normal & $\begin{array}{l}\text { Gastric } \\
\text { ulcer }\end{array}$ & Duodenal ulcer & Gastritis & $\begin{array}{l}\text { p- } \\
\text { value }\end{array}$ \\
\hline sTREM-1 (pg/ml); Median (IQR) & $19(6.75-60.50)$ & $22(10-34)$ & $18.5(10.75-35.25)$ & $22(11.50-44)$ & 0.92 \\
\hline CRP (ng/ml); Mean (SD) & $6.6(3.6)$ & $5.2(2.7)$ & $6.5(2.9)$ & $6.3(3.4)$ & 0.2 \\
\hline
\end{tabular}

sTREM-1, soluble triggering receptor expressed on myeloid cells; CRP, C-reactive protein; SD, standard deviation; $\mathrm{IQR}$; inter quartile range. 


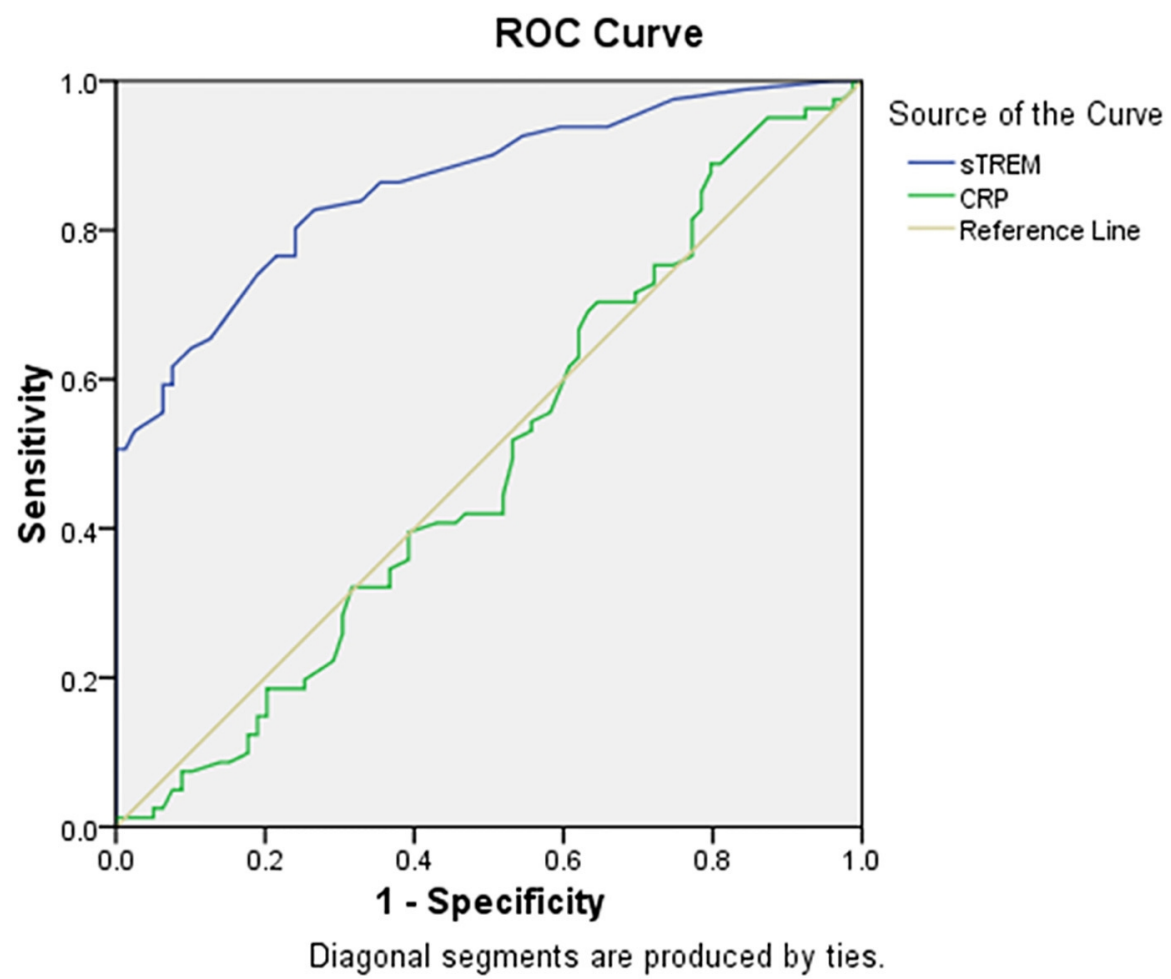

Figure 1. Receiver operating characteristic curve (ROC) for soluble triggering receptor expressed on myeloid cells (sTREM-1) and C-reactive protein (CRP).

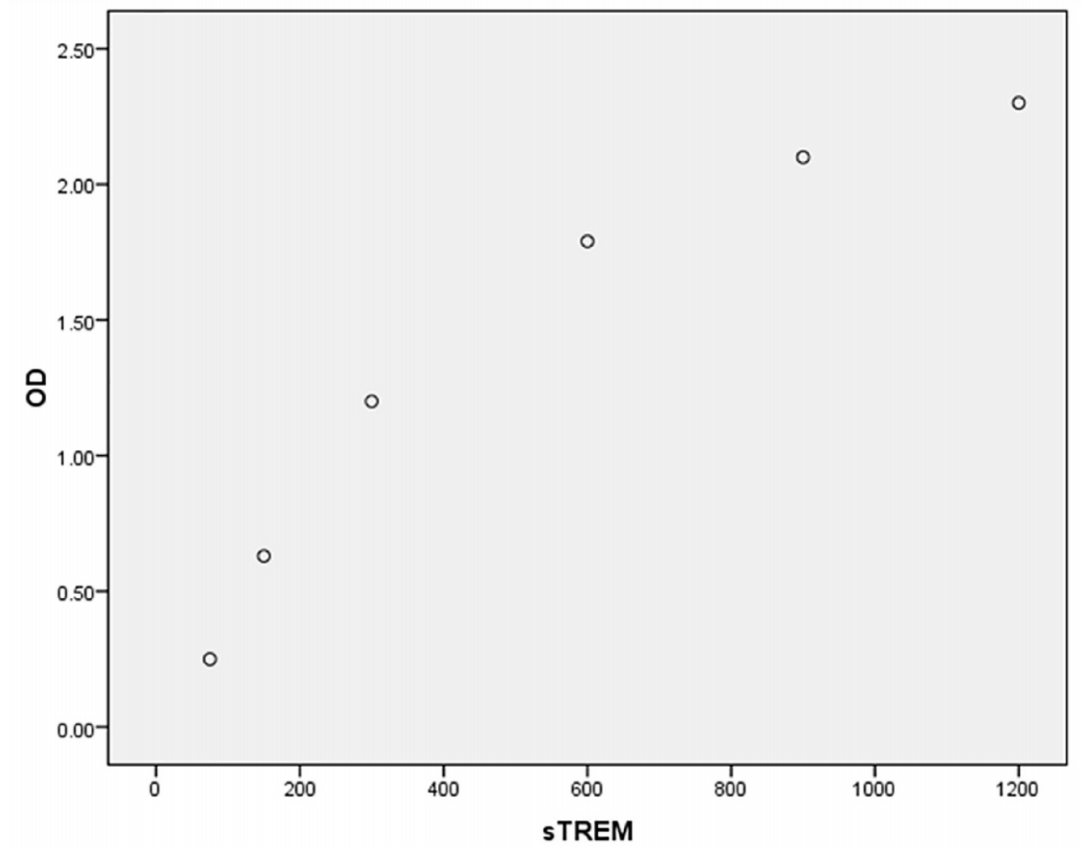

Figure 2. Calibration plot for soluble triggering receptor expressed on myeloid cells (sTREM-1) concentration $(\mathrm{pg} / \mathrm{ml})$ according to optic density (OD). 


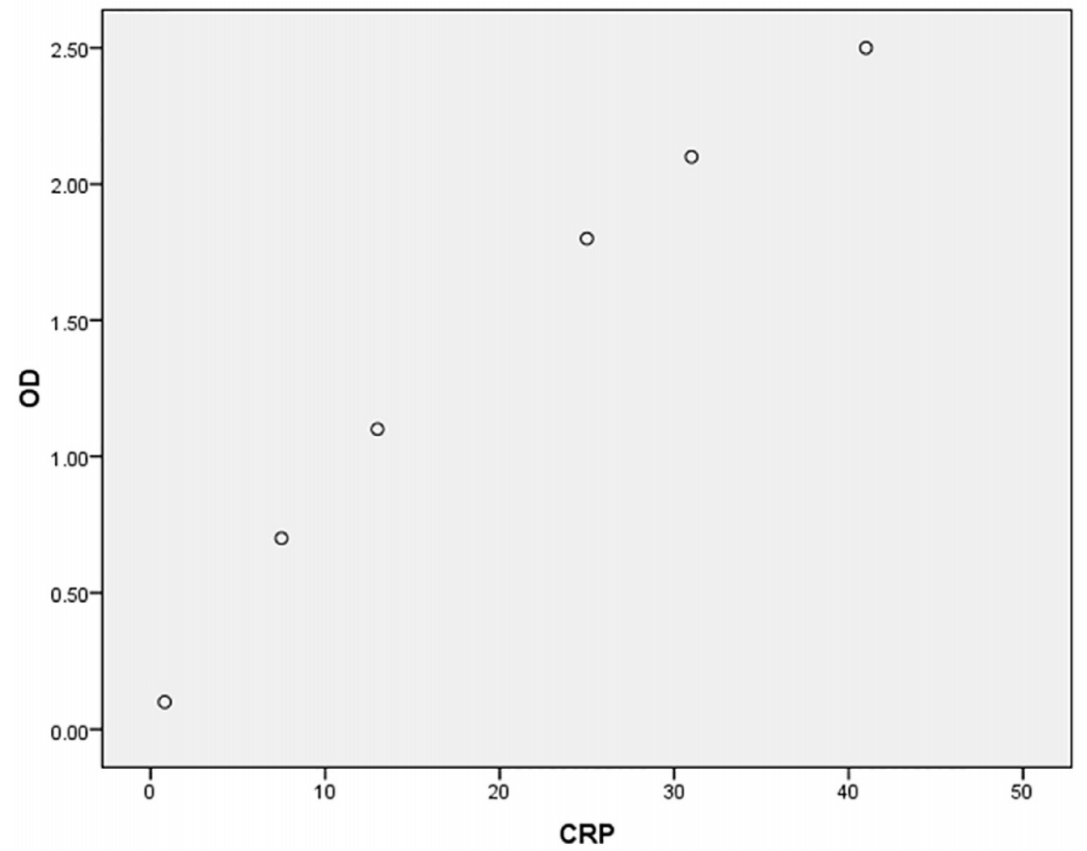

Figure 3. Calibration plot for C-reactive protein (CRP) concentration ( $\mathrm{ng} / \mathrm{ml})$ according to optic density (OD).

\section{Discussion}

The aim of this research was to compare the diagnostic values of gastric juice sTREM-1 and CRP for diagnosis of $H$. pylori infection. The present study revealed that despite CRP, sTREM-1 was a relatively good indicator of $H$. pylori infection of gastric mucosa. In contrast, CRP and sTREM-1 levels were not statistically different according to endoscopic findings in our survey. In an investigation, the mainstay assumption was that sTREM-1 was secreted by recruited phagocytes to inflamed gastric mucosa and eradication of $H$. pylori decreased the level of sTREM- 1 in gastric juice (9) which was somehow compatible with the current investigation. During a survey, sTREM-1 gastric juice level was not related to the infection by $H$. pylori, but was associated with peptic ulcer disease (7), which was inconsistent with the present study. The cause of these differences may be due to various pathogenesis of peptic ulcer disease and chronic gastritis. During an investigation, sTREM-1 was increased in the gastric juice of patients with peptic ulcer disease despite chronic gastritis (20), that was incompatible with our investigation. In another survey, serum sTREM-1 titer was high among patients with peptic ulcer disease and was decreased significantly following treatment especially with complete healing (21). These findings may be due to the hypothesis that sTREM-1 is a probable factor leading to peptic ulcer (20). Su et al. found that sTREM-1 was more reliable than CRP for diagnosis and severity of sepsis (22) which was compatible with our results. Lin et al. indicated that neither sTREM-1 nor CRP were good markers for detection of infection (23), which was not in line with our study. No study has been performed in Iran on gastric juice level of sTREM-1 in patients with peptic ulcer disease and chronic gastritis or as a diagnostic test to detect the gastric infection by $H$. pylori. C-reactive protein, an infection and inflammation marker, is an acute phase reactive protein and is commonly used in detection of early onset neonatal sepsis due to high sensitivity of this test (24). According to our knowledge, CRP was not a good predictor of bacterial infection (H. pylori) in gastric juice with low sensitivity and specificity. Some researches described that CRP was not an appropriate diagnostic tool for detection of sepsis compared to other tests $(25,26)$ which was in line with ours. According to Miedema et al. (27), CRP was indicated a poor diagnostic test for discrimination of bacterial infection with low sensitivity and specificity that was congruent with our results. During an investigation, an acceptable correlation was shown between salivary and serum CRP levels for screening of at-risk neonates for infection (28) which was incompatible with the present study. Stettin et al. indicated that serum CRP level was significantly higher in $H$. pylori infected cases than in the control group (29) which was not in line with our survey. In another research, the mean serum concentration of high-sensitivity CRP (hs-CRP) among individuals with $H$. pylori infection was significantly greater than uninfected cases $(30)$ which was incongruent with our results. No study has been conducted on CRP level of gastric juice for detection of gastric mucosa infection by $\mathrm{H}$. pylori. Our research indicated no association between gastric juice CRP level and infection of gastric mucosa with H. pylori, and the probable cause may be due to gastric juice acidity and many digestive enzymes which confounded our 
results. The strong points of the present study include: 1 - Relatively sufficient sample size. 2- The first research on sTREM-1 level of gastric juice for detection of $H$. pylori infection in Iran. 3- The first study on association between gastric juice CRP level with endoscopic findings among dyspeptic patients and gastric $H$. pylori infection. 4Sensitivity and specificity calculation of sTREM-1 and CRP for detection of gastric H. pylori infection. The limitations of our study include: 1- No neutralization of gastric juice acidity, digestive enzymes and other proteins during measuring the CRP level. 2- We did not indicate the grading of gastritis by histopathology and its relation with H. pylori infection and the levels of sTREM-1 and CRP. 3- We only used histopathology as the gold standard test and due to false negative and positive results, it would be better to use some other modalities such as rapid urea test, PCR, culture or urea breath test besides histopathology.

\section{Conclusions}

Our results indicated that despite CRP, sTREM-1 was a relatively acceptable marker for detection of gastric $H$. pylori infection. The applied issue is that gastric sTREM-1 may be useful for detection of $H$. pylori besides gastric biopsy. It is recommended to measure gastric juice sTREM-1 level by NG tube as a supplementary test accompanied by non-invasive tests such as urea breath test, serologic or stool antigen test to compare its diagnostic values with them. More researches on gastric juice CRP and sTREM-1 levels with more comprehensive laboratory details for diagnosis of $H$. pylori infection will be helpful in the future.

\section{Acknowledgments:}

The authors would like to thank Deputy of Research, Kashan University of Medical Sciences for financial support with grant No. 94108. We are thankful for endoscopy unit staffing and laboratory personnel of Shahid Beheshti Hospital of Kashan for their valuable cooperation in conducting this study.

\section{Conflict of Interest:}

There is no conflict of interest to be declared.

\section{Authors' contributions:}

All authors contributed to this project and article equally. All authors read and approved the final manuscript.

\section{References:}

1) Padol S, Yuan Y, Thabane M, Padol IT, Hunt RH. The effect of CYP2C19 polymorphisms on H. pylori eradication rate in dual and triple first-line PPI therapies: a meta-analysis. Am J Gastroenterol. 2006; 101(7): 1467-75. doi: 10.1111/j.1572-0241.2006.00717.x. PMID: 16863547.

2) Calam J, Baron JH. ABC of the upper gastrointestinal tract: Pathophysiology of duodenal and gastric ulcer and gastric cancer. BMJ. 2001; 323(7319): 980-2. PMID: 11679389, PMCID: PMC1121510.

3) Vakil N. Helicobacter pylori treatment: a practical approach. Am J Gastroenterol. 2006; 101(3): 497-9. doi: 10.1111/j.1572-0241.2006.00454.x. PMID: 16542285.

4) Eckmann L. Sensor molecules in intestinal innate immunity against bacterial infections. Curr Opin Gastroenterol. 2006; 22(2): 95-101. doi: 10.1097/01.mog.0000208458.38772.2a. PMID: 16462163, PMCID: PMC2695762.

5) Bouchon A, Dietrich J, Colonna M. Cutting edge: inflammatory responses can be triggered by TREM-1, a novel receptor expressed on neutrophils and monocytes. J Immunol. 2000; 164(10): 4991-5. PMID: 10799849.

6) Gibot S, Kolopp-Sarda MN, Bene MC, Bollaert PE, Lozniewski A, Mory F, et al. A soluble form of the triggering receptor expressed on myeloid cells- 1 modulates the inflammatory response in murine sepsis. $\mathrm{J}$ Exp Med. 2004; 200(11): 1419-26. doi: 10.1084/jem.20040708. PMID: 15557347, PMCID: PMC2211948.

7) Koussoulas V, Tzivras M, Giamarellos-Bourboulis EJ, Demonakou M, Vassilliou S, Pelekanou A, et al. Can soluble triggering receptor expressed on myeloid cells (sTREM-1) be considered an anti-inflammatory mediator in the pathogenesis of peptic ulcer disease? Dig Dis Sci. 2007; 52(9): 2166-9. doi: 10.1007/s10620-006-9694-z. PMID: 17410457.

8) Koussoulas V, Vassiliou S, Demonakou M, Tassias G, Giamarellos-Bourboulis EJ, Mouktaroudi M, et al. Soluble triggering receptor expressed on myeloid cells (sTREM-1): a new mediator involved in the pathogenesis of peptic ulcer disease. Eur J Gastroenterol Hepatol. 2006; 18(4): 375-9. doi: 10.1097/00042737-200604000-00010. PMID: 16538108.

9) Koussoulas V, Vassiliou S, Spyridaki E, Demonakou M, Vaki I, Barbatzas C, et al. Evidence for the role of gastric mucosa at the secretion of soluble triggering receptor expressed on myeloid cells (strem-1) in peptic 
ulcer disease. World J Gastroenterol. 2007; 13(34): 4610-4. doi: 10.3748/wjg.v13.i34.4610. PMID: 17729416, PMCID: PMC4611837.

10) Bopp C, Hofer S, Bouchon A, Zimmermann JB, Martin E, Weigand MA. Soluble TREM-1 is not suitable for distinguishing between systemic inflammatory response syndrome and sepsis survivors and nonsurvivors in the early stage of acute inflammation. Eur J Anaesthesiol. 2009; 26(6): 504-7. doi: 10.1097/EJA.0b013e328329afca. PMID: 19307971.

11) Gibot S, Le Renard PE, Bollaert PE, Kolopp-Sarda MN, Bene MC, Faure GC, et al. Surface triggering receptor expressed on myeloid cells 1 expression patterns in septic shock. Intensive Care Med. 2005; 31(4): 594-7. doi: 10.1007/s00134-005-2572-x. PMID: 15754199.

12) Phua J, Koay ES, Zhang D, Lee KH. How well do serum sTREM-1 measurements prognosticate in septic shock? Anaesth Intensive Care. 2008; 36(5): 654-8. PMID: 18853582.

13) Rivera-Chavez FA, Minei JP. Soluble triggering receptor expressed on myeloid cells-1 is an early marker of infection in the surgical intensive care unit. Surg Infect (Larchmt). 2009; 10(5): 435-9. doi: 10.1089/sur.2009.030. PMID: 19792836, PMCID: PMC2956524.

14) Cetinkaya M, Ozkan H, Koksal N, Celebi S, Hacimustafaoglu M. Comparison of serum amyloid A concentrations with those of C-reactive protein and procalcitonin in diagnosis and follow-up of neonatal sepsis in premature infants. J Perinatol. 2009; 29(3): 225-31. doi: 10.1038/jp.2008.207. PMID: 19078972.

15) Dillon MC, Opris DC, Kopanczyk R, Lickliter J, Cornwell HN, Bridges EG, et al. Detection of homocysteine and C-reactive protein in the saliva of healthy adults: comparison with blood levels. Biomark Insights. 2010; 5: 57-61. PMID: 20703322, PMCID: PMC2918353.

16) Ouellet-Morin I, Danese A, Williams B, Arseneault L. Validation of a high-sensitivity assay for C-reactive protein in human saliva. Brain Behav Immun. 2011; 25(4): 640-6. doi: 10.1016/j.bbi.2010.12.020. PMID: 21236331.

17) Lacaze-Masmonteil T, Rosychuk RJ, Robinson JL. Value of a single C-reactive protein measurement at 18 h of age. Arch Dis Child Fetal Neonatal Ed. 2014; 99(1): F76-9. doi: 10.1136/archdischild-2013-303984. PMID: 24008814.

18) Ishida $Y$, Suzuki K, Taki K, Niwa T, Kurotsuchi S, Ando H, et al. Significant association between Helicobacter pylori infection and serum C-reactive protein. Int J Med Sci. 2008; 5(4): 224-9. PMID: 18695743, PMCID: PMC2500148.

19) Saribas S, Kocazeybek B, Aslan M, Altun S, Seyhun Y, Oner YA, et al. Do procalcitonin and C-reactive protein levels have a place in the diagnosis and follow-up of Helicobacter pylori infections? J Med Microbiol. 2004; 53(Pt 7): 639-44. doi: 10.1099/jmm.0.05398-0. PMID: 15184535.

20) Schenk M, Bouchon A, Birrer S, Colonna M, Mueller C. Macrophages expressing triggering receptor expressed on myeloid cells-1 are underrepresented in the human intestine. J Immunol. 2005; 174(1): 517 24. doi: 10.1097/00003246-200204000-0000.6. PMID: 15611278.

21) Koussoulas V, Giamarellos-Bourboulis EJ, Barbatzas C, Pimentel M. Serum sTREM-1 as a Surrogate Marker of Treatment Outcome in Patients with Peptic Ulcer Disease. Dig Dis Sci. 2011; 56(12): 3590-5. doi: 10.1007/s10620-011-1761-4. PMID: 21633832. .

22) Su L, Feng L, Song Q, Kang H, Zhang X, Liang Z, et al. Diagnostic value of dynamics serum sCD163, sTREM-1, PCT, and CRP in differentiating sepsis, severity assessment, and prognostic prediction. Mediators Inflamm. 2013; 2013: 969875. doi: 10.1155/2013/969875. PMID: 23935252, PMCID: PMC3713373.

23) Lin CH, Hsieh SC, Keng LT, Lee HS, Chang HT, Liao WY, et al. Prospective Evaluation of Procalcitonin, Soluble Triggering Receptor Expressed on Myeloid Cells-1 and C-Reactive Protein in Febrile Patients with Autoimmune Diseases. PLoS One. 2016; 11(4): e0153938. doi: 10.1371/journal.pone.0153938. PMID: 27096761, PMCID: PMC4838219.

24) Hofer N, Zacharias E, Muller W, Resch B. An update on the use of C-reactive protein in early-onset neonatal sepsis: current insights and new tasks. Neonatology. 2012; 102(1): 25-36. doi: 10.1159/000336629. PMID: 22507868.

25) Claeys R, Vinken S, Spapen H, ver Elst K, Decochez K, Huyghens L, et al. Plasma procalcitonin and Creactive protein in acute septic shock: clinical and biological correlates. Crit Care Med. 2002; 30(4): 757 62. PMID: 11940741.

26) Simon L, Gauvin F, Amre DK, Saint-Louis P, Lacroix J. Serum procalcitonin and C-reactive protein levels as markers of bacterial infection: a systematic review and meta-analysis. Clin Infect Dis. 2004; 39(2): 20617. doi: 10.1086/421997. PMID: 15307030. 
27) Miedema KG, de Bont ES, Elferink RF, van Vliet MJ, Nijhuis CS, Kamps WA, et al. The diagnostic value of CRP, IL-8, PCT, and sTREM-1 in the detection of bacterial infections in pediatric oncology patients with febrile neutropenia. Support Care Cancer. 2011; 19(10): 1593-600. doi: 10.1007/s00520-010-0987-6. PMID: 20803037, PMCID: PMC3166608.

28) Iyengar A, Paulus JK, Gerlanc DJ, Maron JL. Detection and potential utility of C-reactive protein in saliva of neonates. Front Pediatr. 2014; 2: 131. doi:10.3389/fped.2014.00131. PMID: 25485262, PMCID: PMC4239436.

29) Stettin D, Waldmann A, Strohle A, Hahn A. Association between Helicobacter pylori-infection, C-reactive protein and status of B vitamins. Adv Med Sci. 2008; 53(2): 205-13. doi: 10.2478/v10039-008-0050-8. PMID: 19230307.

30) Jafarzadeh A, Hassanshahi GH, Nemati M. Serum levels of high-sensitivity C-reactive protein (hs-CRP)in Helicobacter pylori-infected peptic ulcer patients and its association with bacterial CagA virulence factor. Dig Dis Sci. 2009; 54(12): 2612-6. doi: 10.1007/s10620-008-0686-z. PMID: 19160050. 